\title{
Bullous systemic lupus erythematosus in a 10-year-old child*
}

\author{
Nelise Ritter Hans-Bittner ${ }^{1}$ \\ Günter Hans Filho ${ }^{1,2}$
}

\author{
Guilherme Canho Bittner ${ }^{1}$ \\ Luiz Carlos Takita ${ }^{1,3}$
}

DOI: http:/ /dx.doi.org/10.1590/abd1806-4841.20176074

\begin{abstract}
Bullous systemic lupus erythematosus (BSLE) is a rare autoimmune subepidermal blistering disease, with few cases described in childhood. It has different clinical-pathological features. We report a case of BSLE in a 10-year-old child with systemic lupus erythematosus, treated with prednisone and hydroxychloroquine. There was complete remission with dapsone, with no recurrence of skin lesions throughout one year of follow-up. We highlight the rarity and early age of occurrence.
\end{abstract}

Keywords: Autoimmunity; Skin diseases, vesiculobullous; Lupus erythematosus, systemic

\section{INTRODUCTION}

Bullous systemic lupus erythematosus (BSLE) is a rare cutaneous manifestation of systemic lupus erythematosus (SLE), and even more uncommon in the pediatric population ${ }^{1,2}$ It is a heterogenous disease, caused by autoantibodies against the dermo-epidermal junction, particularly type VII collagen. ${ }^{3}$ Occasionally, other autoantigens, such as laminin 5, laminin 6 and BP230 are described. ${ }^{4}$ BSLE presents a distinct combination of clinical, histological, immunopathological and electron microscopy features. ${ }^{5}$ We report a case of BSLE in a 10-year-old child with SLE, under immunosuppressive treatment with prednisone and hydroxychloroquine for 4 months. We highlight the rarity, due to the fact that it developed during childhood, in a patient under standard SLE treatment.

\section{CASE REPORT}

A 10-year-old female patient, weighing $31 \mathrm{~kg}$ was being followed for SLE over the previous 4 months, on prednisone $(60 \mathrm{mg}$ / day) and hydroxychloroquine ( $130 \mathrm{mg} /$ day). She developed tense, isolated and grouped vesiculobullous lesions on the face, neck, axillae, upper limbs, inguinal region, buttocks and oral, nasal and genital mucosa, over 1 week (Figures 1 to 3). She also had occasional fever and hepatomegaly.
Laboratory tests revealed mild anemia (Hb.: 11.8), CRP 48, hypocomplementemia [C3: $50 \mathrm{mg} / \mathrm{dL}$ (90 mg/dL to $180 \mathrm{mg} / \mathrm{dL}$ ) and C4: $14 \mathrm{mg} / \mathrm{dL}$ (19 mg/dL to $52 \mathrm{mg} / \mathrm{dL})]$ and ANA 1/640 in a homogenous nuclear pattern, besides positive anti-dsDNA, P-ANCA, anti-Ro and lupus anticoagulant. Urinalysis and renal function were normal, and proteinuria was slightly increased (184 mg/24 hours). Echocardiogram showed a minimal increase in pericardial fluid and chest radiography showed a mild, bilateral pleural effusion.

Histology from skin biopsy revealed subepidermal blister filled with fibrin, lymphomononuclear cells and neutrophils, thickening of the basement membrane in the dermal papillae and mild perivascular lymphoplasmacytic infiltrate in the papillary, superficial dermis (Figure 4). Direct immunofluorescence (DIF) showed deposition of linear IgG, IgA and C3 in the basement membrane zone and granular IgM (Figure 5). Salt-split was positive for IgG in the dermis and epidermis and for IgA in the epidermis. Indirect immunofluorescence was negative for IgA, IgM and IgG, but the nuclei of keratinocytes fluoresced (Figure 6).

Clinical and laboratory abnormalities in this patient confirmed the suspicion of BSLE. We maintained the previous treat-

Study submitted on 22.05.2016

Approved by the Advisory Board and accepted for publication on 19.09.2016

* Study conducted at Dr. Günter Hans Department of Dermatology, at Hospital Universitário Maria Aparecida Pedrossian, of the Universidade Federal de Mato Grosso do Sul (HUMAP-UFMS) - Campo Grande (MS), Brazil.

Financial support: None

Conflict of interest: None

1 Dr. Günter Hans Department of Dermatology at Hospital Universitário Maria Aparecida Pedrossian, of the Universidade Federal de Mato Grosso do Sul (HUMAP-UFMS) - Campo Grande (MS), Brazil.

2 Discipline of Dermatology of the Faculdade de Medicina, Universidade Federal de Mato Grosso do Sul (FAMED-UFMS) - Campo Grande (MS), Brazil.

3 Discipline of Pathology of the Faculdade de Medicina, Universidade Federal de Mato Grosso do Sul (FAMED-UFMS) - Campo Grande (MS), Brazil. 
ment (prednisone and hydroxychloroquine), associating dapsone $(1,5 \mathrm{mg} / \mathrm{kg} /$ day $)$, with complete remission of the cutaneous lesions. The patient remained stable during 1 year of follow-up.

\section{DISCUSSION}

BSLE is a rare bullous variant, ${ }^{1,3-6}$ affecting less than $5 \%$ of SLE patients. ${ }^{6}$ It is even more uncommon in the pediatric population and presents rarely as the initial manifestation of lupus erythematosus. ${ }^{6}$ In our case, the bullous disease manifested 4 months after the diagnosis and commencement of treatment for SLE.

Clinically, BSLE is characterized by a generalized vesiculobullous eruption, non-cicatricial, restricted to photo-exposed areas,

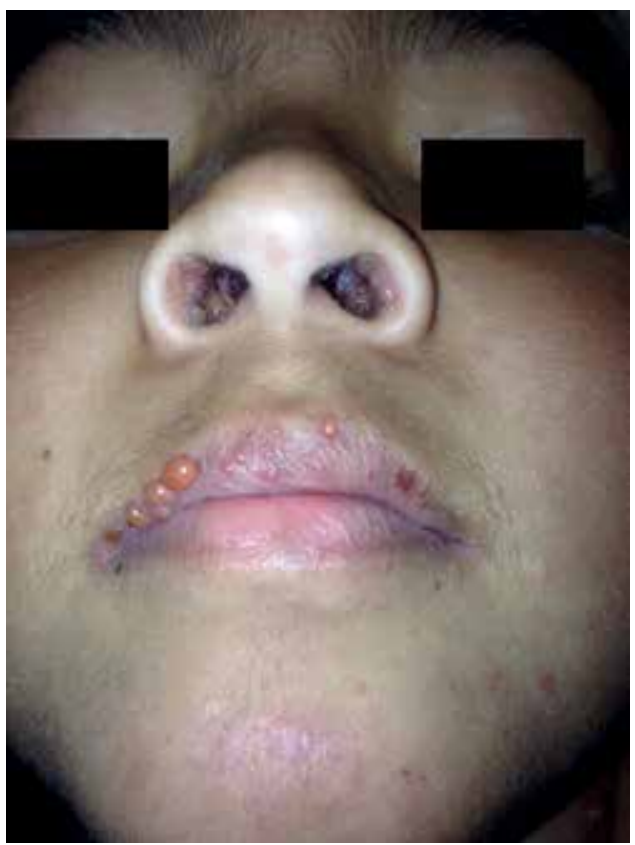

Figure 1: Scattered tense vesicles/bullae on the nasal and labial mucosa

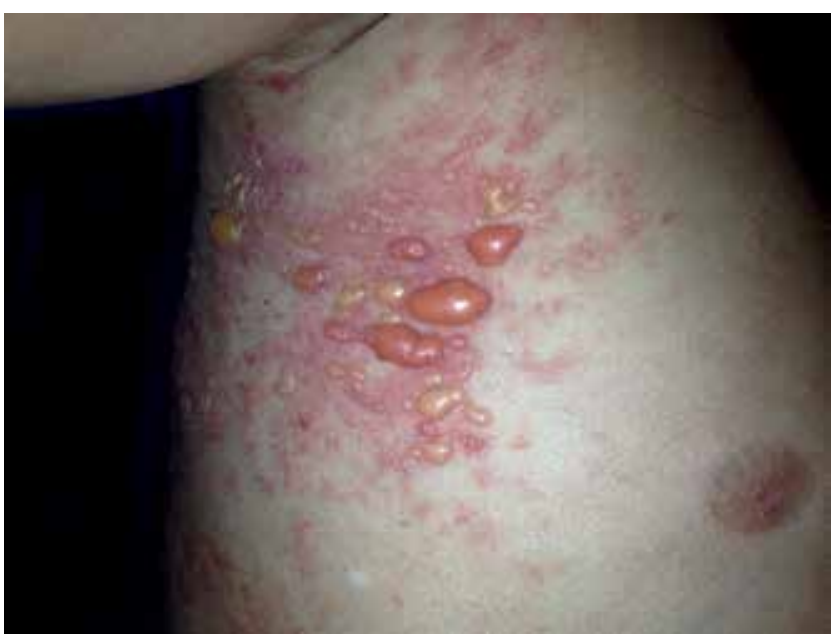

Figure 2: Tense bullae overlying an erythematous base on the right axilla or not. On histology, a subepidermal blister with neutrophilic inflammatory infiltrate is seen. DIF demonstrates a linear or granular deposition of IgG, IgA and C3 in the basement membrane zone., ${ }^{1,5}$

BSLE diagnostic criteria were initially proposed by Camisa and Sharma and posteriorly revised by Gammon and Briggaman, that included autoantibodies against collagen type VII. ${ }^{2}$ The criteria are: 1) fulfillment of the criteria for SLE by the American College of Rheumatology; 2) acquired non-cicatricial bullous eruption in, but not limited to, sun-exposed areas; 3) subepidermal blisters with a predominantly neutrophilic infiltrate in the dermis and basement membrane zone; and 4) deposition of IgG, IgA, IgM and C3 on DIF of perilesional skin. Circulating autoantibodies against collagen type VII, confirmed by indirect salt-split skin or immunoprecipitation and immunoglobulin deposits (Igs) in the same distribution of anchoring fibrils/collagen type VII on electronic microscopy corroborate autoimmunity against collagen type VII. ${ }^{7}$

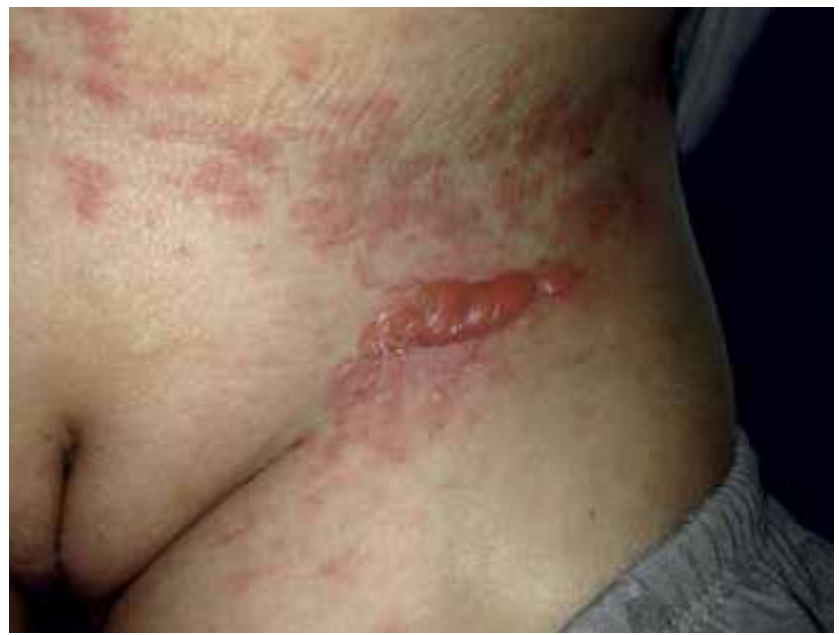

Figure 3: Involvement of the left inguinal region and vaginal introitus

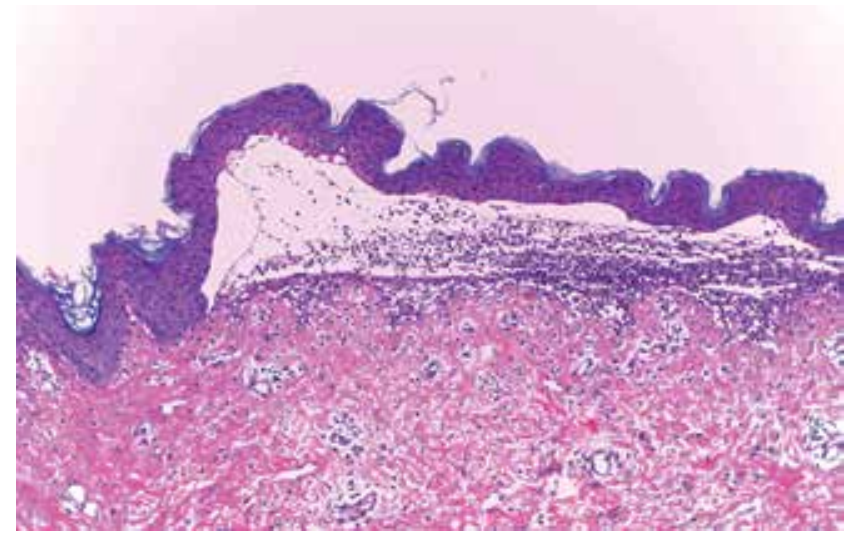

Figure 4: Subepidermal blister filled with fibrin, lymphomononuclear and neutrophils. Basement membrane thickening (Hematoxylin \& eosin, X100) 


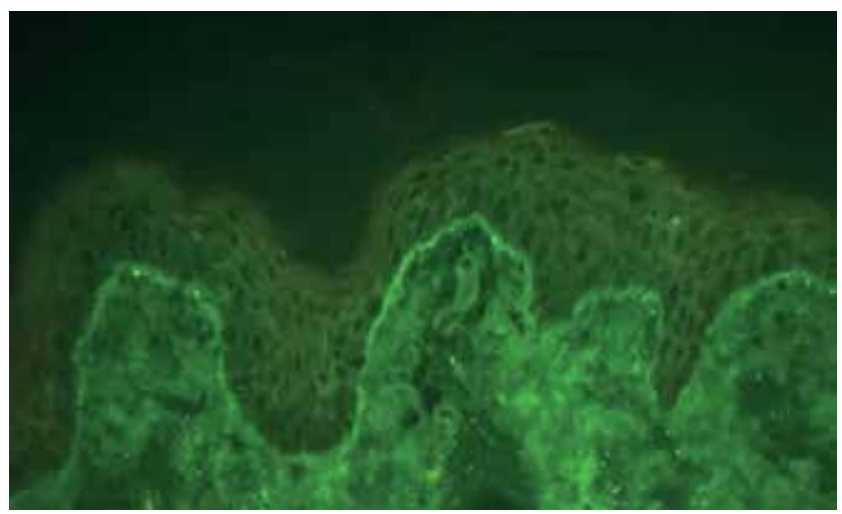

Figure 5: Direct immunofluorescence with linear deposits of C3 in the BMZ

In our case, the patient being treated for SLE presented with mostly tense bullae, mainly on the face, mucosa and flexures. Histopathology revealed findings suggestive of BSLE and DIF showed deposits of $\operatorname{IgG}, \operatorname{IgA}$, IgM and C3 in the basement membrane zone - complete lupus band -, thus confirming the diagnosis of BSLE.

The activity of the bullous disease can occur isolated from or concomitantly with other systemic manifestations of SLE, nephritis and serositis in particular. ${ }^{1,2}$ Therefore, in all cases of BSLE, we should always monitor systemic changes, as in SLE. ${ }^{5}$ In our case, raised levels of antinuclear antibodies, hypocomplementemia, anemia, mild proteinuria and pleural and pericardial effusion were seen, indicating active SLE and emphasizing the role of the dermatologist in the identification and early diagnosis of these abnormalities.

The main differential diagnoses of BSLE are acquired epidermolysis bullosa, dermatitis herpetiformis, bullous pemphigoid and linear IgA bullous dermatosis. ${ }^{5}$ However, the clinical presen-

\section{REFERENCES}

1. Barbosa WS, Rodarte CM, Guerra JG, Maciel VG, Fleury Júnior LF, Costa MB. Lúpus eritematoso sistêmico bolhoso - diagnóstico diferencial com dermatite herpetiforme. An Bras Dermatol. 2011;86:S92-5.

2. Lourenço DM, Gomes RC, Aikawa NE, Campos LM, Romiti R, Silva CA. Childhoodonset bullous systemic lupus erythematosus. Lupus. 2014;23:1422-5.

3. Contestable JJ, Edhegard KD, Meyerle JH. Bullous systemic lupus erythematosus: a review and update to diagnosis and treatment. Am J Clin Dermatol. 2014;15:517-24.

4. Grover C, Khurana A, Sharma S, Singal A. Bullous Systemic Lupus Erythematosus. Indian J Dermatol. 2013;58:492.

5. Chiminazzo MD, Cardoso G, Pegas JRP, Aoki V, Sanchez APG. Lúpus eritematoso sistêmico bolhoso na infância - Relato de caso. An Bras Dermatol. 2007;82:257-61.

6. Tincopa M, Puttgen KB, Sule S, Cohen BA, Gerstenblith MR. Bullous lupus: an unusual initial presentation of systemic lupus erythematosus in an adolescent girl. Pediatr Dermatol. 2010;27:373-6.

7. Gammon WR, Briggaman RA. A phenotypically distinctive but immunologically heterogeneous bullous disorder. J J Invest Dermatol. 1993;100:28S-34S.

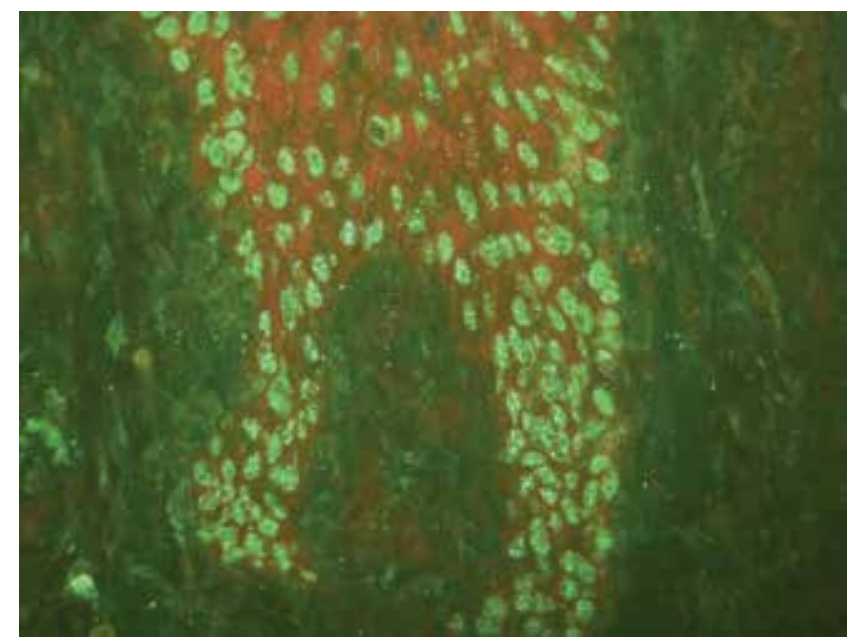

Figure 6: Direct immunofluorescence with fluorescence of the keratinocyte nuclei

tation, combined with the histology, immunologic tests and other diagnostic methods can distinguish BSLE from these conditions. ${ }^{3}$

The treatment of choice is dapsone; the therapeutic response is usually satisfactory. ${ }^{1,4}$ If necessary, prednisone and, eventually, azathioprine, cyclophosphamide or methotrexate can be used. ${ }^{1}$ Recently, the use of rituximab has been shown to be effective in refractory cases. ${ }^{3}$ In this patient, the BSLE lesions appeared despite the use of antimalarial (hydroxychloroquine) and immunosuppressive doses of prednisone $(2 \mathrm{mg} / \mathrm{kg} /$ day), supporting the opinion of some authors that affirm that BSLE is relatively resistant to steroids and other immunosuppressants. ${ }^{4}$ In this case, we opted to maintain the dose of the previous treatment and add dapsone, obtaining a favorable clinical response, with no evidence of bullous lesions in 1 year of follow-up.

The reported case describes a florid BSLE in childhood, with emphasis on the patient's age besides its appearance during standard SLE treatment.]

\section{MAILING ADDRESS: \\ Nelise Ritter Hans Bittner \\ Rua Princesa Isabel, 422 \\ Jardim dos Estados \\ 79002-510 Campo Grande, MS \\ Brazil \\ E-mail:nelisehans@gmail.com}

How to cite this article: Hans-Bittner NR, Bittner GC, Hans-Filho G, Takita LC. Bullous systemic lupus erythematosus in a 10-year-old child. An Bras Dermatol. 2017;92(5 Suppl 1): 37-9. 